\title{
High PON1 Expression Predicts Poor Prognosis In Kidney Renal Clear Cell Carcinoma
}

\section{Chenxia Jiang}

Affiliated Hospital of Nantong University

\section{Xinyu Zhang}

Affiliated Hospital of Nantong University

Xiaoyan Li

Affiliated Hospital of Nantong University

Jia Li

Tongling People's Hospital

Hua Huang ( $\nabla$ ntfyhuanghua@163.com )

Affiliated Hospital of Nantong University

\section{Research Article}

Keywords: PON1, Kidney renal clear cell carcinoma, Prognosis, Overall survival, Immunity

Posted Date: December 15th, 2021

DOl: https://doi.org/10.21203/rs.3.rs-1112733/v1

License: (c) (i) This work is licensed under a Creative Commons Attribution 4.0 International License. Read Full License 


\title{
High PON1 expression predicts poor prognosis in kidney renal clear cell carcinoma
}

Chenxia Jiang ${ }^{1^{*}}$, Xinyu Zhang ${ }^{2 *}$, Xiaoyan $\mathrm{Li}^{3^{*}}$, Jia $\mathrm{Li}^{4 \star}$, Hua Huang ${ }^{1 \star}$

${ }^{1}$ Department of Pathology, Affiliated Hospital of Nantong University, Nantong, 226001, Jiangsu Province, China.

${ }^{2}$ Department of Urology, Affiliated Hospital of Nantong University, Nantong, 226001, Jiangsu Province, China.

${ }^{3}$ Department of Imformation, Affiliated Hospital of Nantong University, Nantong, 226001, Jiangsu Province, China.

${ }^{4}$ Department of Pathology, Tongling People's Hospital, Tongling, 244000, Anhui Province, China.

"Chenxia Jiang, Xinyu Zhang and Xiaoyan Li contributed equally to this work.

${ }^{\star}$ Corresponding author:

Hua Huang

Department of Pathology, Affiliated Hospital of Nantong University, No.20 West Temple Road, Nantong, 226001, Jiangsu Province, China.

E-mail: ntfyhuanghua@163.com

TEL: +08613815209685

Jia Li

Department of Pathology, Tongling People's Hospital, Tongling, 244000, Anhui Province, China. E-mail: lijia543144676@126.com

TEL: +08613685629065

\begin{abstract}
Background: Relevant study had demonstrated that Paraoxonase-1 (PON1) had relationship with occurrence and development of tumors which suggested that PON1 was a key gene in promoting
\end{abstract}


tumor progression. However, the relationship between PON1 and Kidney renal clear cell carcinoma (KIRC) is still unclear so far.

Methods: We downloaded relevant data about KIRC from TCGA dataset and compared it with normal renal tissues. Immunohistochemistry (IHC) was applied to analyze the expression of PON1. Univariate cox regression analysis and multivariate cox regression analysis were also utilized to analyze independent factors associated with prognosis. Gene set enrichment analysis was conducted to find the signaling pathways of PON1 in KIRC. Finally, we also investigated whether PON1 had relationship with immunity.

Results: As shown in results, PON1 expression was decreased in KIRC compared with adjacent paracancer tissues. Immunohistochemistry (IHC) was utilized to find the expression of PON1. After survival analysis, the high expression of PON1 was significantly related to overall survival $(\mathrm{P}<0.001)$. Univariate/Multivariate cox regression analysis both revealed that PON1 could serve as an independent prognostic factor. To analyze overall survival (OS) of patients with KIRC, nomogram was developed. GSEA revealed that PON1 was correlated with homologous recombination. Besides, PON1 had few relationships with immunity.

Conclusions: Our results revealed that PON1 could serve as an independent prognostic factor for KIRC, providing a novel target for KIRC future treatments.

Keywords: PON1, Kidney renal clear cell carcinoma, Prognosis, Overall survival, Immunity

\section{Introduction}

Kidney renal clear cell carcinoma (KIRC) is one of the most common tumor diseases of the urinary system, accounting for $2 \%$ to $3 \%$ of adult malignancies (1). An estimated 76080 new cases 
of kidney cancer and an estimated 13780 deaths will occur in 2021(1). KIRC often has three main clinical manifestations which goes undetected early (2). Due to the lack of typical clinical manifestations, some kidney cancer patients was found to have metastasized by the time the tumor was found(3). Surgery and chemoradiotherapy are conventional treatments for KIRC. However, patients with distant metastasis have a low survival rate. Besides, distant metastasis of tumor can greatly affect the prognosis of patients. Therefore, we hope to improve the prognosis of KIRC patients by explore pathogenesis of PON1.

Paraoxonase-1 (PON1), located at chromosome 7 (q21.22) is a member of a gene family which also contains PON2 and PON3(4). PON1 bound to circulating HDL particles is known to enzymatic hydrolysis of organic sulfates involved in lipid metabolism in disease. The expression of PON1 is related to the formation of atherosclerosis. PON1 can interfere with the formation of atherosclerosis by inhibiting lipid oxidation and reducing the production of lipid peroxides $(5,6)$. More and more studies have revealed that PON1 plays a significant role in the progression of diabetes, atherosclerosis and cancer (7-9). Studies have found that some PON1 genotypes may be a risk factor for cancer, so the role of PON1 in cancer disease models can be predicted(10). In this study, we carried out GSEA to explore relevant signaling pathways and revealed the relationship between PON1 and immunity.

\section{Method and materials}

\subsection{Acquisition and preparation of data}

We obtained Gene expression profiles and related clinical information in regard to KIRC from The Cancer Genome Atlas (TCGA) Data Portal (https://tcga-data.nci.nih.gov/tcga/; accessed October 2021). The differently expressed genes (DEGs) were acquired by applying the "limma" package in view of $\mid \log 2$ fold change (FC) $\mid \geq 1$ and false discovery rate (FDR) $<0.05$. All of these were operated by the $\mathrm{R}$ software (https://www.r-project.org/). Batch normalization of gene expression data were carried out by using the "sva" package in R software.

\subsection{Immunohistochemistry staining}

Tissue sample of KIRC fixed with formalin were used for Immunohistochemistry staining. PON1 antibodies for immunohistochemical staining were obtained from Abcam (ab92466). We 
compared the staining between renal carcinoma and normal tissue under the microscope. The final scores depended on two kinds of factors: positive cells score and staining intensity score. Firstly, positive cell score: $0-5 \%$ is $0,6-25 \%$ is $1,26-50 \%$ is $2,51-75 \%$ is 3 and greater than $75 \%$ is 4 . Besides, score of staining intensity: 0 for no staining, 1 for a slightly yellow background, 2 for a yellowish background and 3 for a brown background. Samples were collected from kidney cancer patients at the Affiliated Hospital of Nantong University. The ethical review was passed by the Affiliated Hospital of Nantong University. We performed immunohistochemical tests on 10 renal cancers tissue and 10 paracancerous tissues.

\subsection{Gene set enrichment analysis}

Gene set enrichment analysis (GSEA) is a scientific method that was used to elucidate the difference in prognosis between patients with the high- and low-PON1 groups. Differentially expressed PON1 was performed KEGG enrichment analysis by using R software. GSEA was used to investigate PON1-related signaling pathways. It would be significant that only the normalized enrichment score (NES) $>1.5$ and nominal $p$ value $<0.05$.

\subsection{Univariate and multivariate Cox hazard regression analysis}

With the help of R software, we used univariate and multivariate COX hazard regression analysis to estimate whether these clinical factors (age, gender, grade, T, M, N, race, stage) and the expression of PON1 were independent prognostic factors.

\subsection{The development of Nomogram}

A nomogram model was conducted to predict the OS of KIRC patients by using "rms" package of R software. The nomogram model included nine independent factors which was used to calculate the total points. Finally, the total point was used to assess OS of KIRC patients.

\subsection{PPI, MSI, TMB and neoantigen}

PPI network was constructed by using the STRING website(http://string-db.org). Then the relationships between these proteins were represented by using Cytoscape3.7.0 software. PPI network analysis was used to reveal the potential relationship between PON1 and other genes. Microsatellite instability (MSI) is defined as the high polymorphism on the length of microsatellites caused by abnormalities in the MMR system (11). We explored the correlation between PON1 expression and MSI, TMB, neoantigen by using the Spearman's method $(12,13)$. The above researches were conducted by Sangerbox tools (http://www.sangerbox.com/tool).

2.7. The immune infiltrations, immune checkpoint molecules, tumor microenvironment and immune cells pathway 
Tumor Immune Estimation Resource (TIMER) was utilized to investigate molecular characterization of tumor-immune interactions (14). TIMER calculated the levels of tumor-infiltrating immune cells of six subtypes which includes B cell, CD4+ T cell, CD8+ T cell, neutrophil, macrophage, and dendritic cell in 10897 tumors of 32 cancer types $(14,15)$. By using the ESTIMATE algorithm, the ImmuneScore, StromalScore and ESTIMATEScore were calculated to analyze tumor microenvironment (16). CIBERSORT which is computational deconvolution method was utilized to explore the immune infiltrations with $\mathrm{P}<0.001$ as standard. We used the Sangerbox tools (http://www.sangerbox.com/tool) to analyze immune checkpoint molecules or immune cells.

\section{Results}

\subsection{Different expression of PON1 and survival outcome}

As shown in Figure.1(A-C), the expression of PON1 in normal tissue is higher than the expression of PON1 in KIRC. These results suggest that the expression of PON1 was significantly decreased compared to adjacent paracancer tissues. The expression of PON1 was also different in READ, PAAD, LUAD, LGG and ESCA. As shown in Fig.1D, Kaplan-Meier survival analysis showed that high-PON1 groups had a worse OS than that low-PON1 groups $(\mathrm{P}<=0.001)$. Increased expression of PON1 in KIRC predicted poor prognosis. We used data downloaded from GSE14994 to conduct the validation (Fig.1E). Among the data, there were 11patients in the normal group and 59 patients in the tumor group.

\subsection{Association with PON1 expression and clinicopathologic variables}

Clinicopathological data were integrated with PON1 expression levels. Logistic regression analysis was applied to analyze the association between PON1 expression and clinicopathologic characteristics in KIRC patients. High PON1expression is significantly related to grade $(\mathrm{P}=0.019)$, $\mathrm{M}(\mathrm{P}=0.019)$, clinical stage $(\mathrm{P}=0.0014)$ and $\mathrm{T}(\mathrm{P}=0.0063)$ (Fig.2). These results all suggested that KIRC patients with high PON1 expression were prone to an advanced form of the diseases and worse status than those with low PON1 expression. Otherwise, Immunohistochemistry (IHC) analysis staining results was expressed highly in tumor tissue compared to normal tissue (Fig.3).

\subsection{PON1 can serve as an independent prognostic marker in KIRC patients}


We used univariate/multivariate Cox regression analyses to verify whether PON1 was an independent prognostic factor by the TCGA dataset (Table1). As shown in univariate Cox analysis, age, grade, stage, T, M and high PON1 expression were significantly related to OS for KIRC $(\mathrm{p}<0.05)$. Besides, multivariate Cox regression analysis revealed that age, grade, stage and PON1 expression $(\mathrm{p}<0.05)$ were also independent risk factors for OS. Above all, these results all demonstrated that PON1 could serve as an independent prognostic marker in KIRC patients.

\subsection{Establishment of the nomogram}

To further predict the 1-year survival, 3-year survival, 5-year survival of the KIRC patients, we developed the nomogram (Fig.4). The nomogram included eight clinicopathological characteristics and PON1 gene. As shown in Figure.4A, age, grade, stage and PON1 were independent factors related to OS. Nevertheless, age, grade, stage, T, M and PON1 expression were significantly related to OS (Figure.4B). We calculated total points by figure out individual point of KIRC patients. Then, we estimated the 1-, 3-, 5-year survival of the KIRC patients.

\subsection{GSEA identifies a PON1-related signaling pathway}

In order to further figure out the pathogenesis of PON1 in KIRC, we carried out GSEA. We made a comparison between the high- and low PON1 expression datasets. As shown in Fig.5A, homologous recombination is enriched in PON1 low expression phenotype. GSEA helped us have a better comprehension of the pathogenesis.

\subsection{PPI, MSI, TMB, Neoantigen construction}

To further investigated the correlations between PONland other genes in KIRC, we generated the PPI network by using the STRING website(http://string-db.org)(Fig.5C). Based on the expression data of renal carcinoma in TCGA, we obtained MSI, TMB and Neoantigen construction (17) .We also conducted an analysis whether PON1 was related to MSI, TMB and Neoantigen construction based on the data of the KIRC patients. Finally, we found that PON1 was not related to MSI, TMB and Neoantigen construction (Fig.5B).

\subsection{The immune infiltrations and tumor microenvironment of KIRC}

In order to find the relationships between PON1 and immunity, we analyzed the immune infiltrations, tumor microenvironment based on the TIMER. As shown in Fig.6A, PON1 was only correlated with neutrophil infiltration $(\mathrm{p}=0.000775)$. The same for the tumor microenvironment, 
PON1 was not linked to StromalScore, ImmuneScore and ESTIMATEScore (Fig.6B).

\subsection{Correlation between PON1 and immune checkpoint molecules, immune cells}

Based on the expression data of KIRC in TCGA, we calculated the relationship between PON1 and immune microenvironment (17). Finally, we found that PON1 was not associated with immune checkpoint molecules and immune cell pathway. After the analysis of the relationship between PON1 and immune checkpoint molecules, we known that there were no significant molecules (Fig7.A). As shown in Fig.7A, the immune checkpoint molecules were not associated with PON1. Besides, we also investigated the relationship between immune cell pathways and PON1. PON1 was only linked to the monocyte pathway (Fig.7B)

\section{Discussion}

Renal cell carcinoma has always been a relatively common tumor in the urinary system. Hematuria, lumbago and abdominal mass are the main clinical manifestations of the renal cell carcinoma. Besides, KIRC serve as the most common pathological phenotype of renal carcinoma. Some studies have demonstrated that the development of KIRC have something to do with the loss or mutation of VHL tumor suppressor gene (18). Surgery is the best treatment for early kidney renal clear cell carcinoma. Relevant meta-analysis studies have revealed that targeted therapy and chemotherapy both have a role to play in KIRC (19). However, more KIRC patients has shown resistance to radiotherapy and chemotherapy (20). More and more study confirmed that PON1 was a key gene in promoting tumor progression. Therefore, it is imperative to analyze the effect of PON1 on KIRC, so as to further improve the prognosis of patients with KIRC.

This article was mainly to explore the correlations among OS of KIRC and the PON1 expression. According to the information of KIRC patients downloaded from the TCGA database, we found that the expression of PON1 in tumor tissues was lower than that in adjacent paracancer tissues. In addition, referring to logistic regression analysis, we found that the high expression of PON1 was significantly related to overall survival in KIRC patients. High-throughput RNA sequencing data indicated that the high expression of PON1 was associated with some clinicopathological features of advanced renal clear cell carcinoma such as age, grade, stage, $\mathrm{T}$ and M. In addition, referring to immunohistochemistry, we confirmed that PON1 had a high 
expression in KIRC. Univariate/Multivariate COX regression analysis further revealed that PON1 could serve as an independent prognostic factor for KIRC. All in all, these mentioned results revealed that PON1 may be a potential independent prognostic factor for KIRC.

As shown in previous studies, PON1 was involved in lipid metabolism and has antioxidant capacity. Therefore, PON1 could reduce LDL-related lipid accumulation(21). Recent researches had proven that PON1 had a great influence on the progression of diabetes, atherosclerosis and cancer $(7,9,22)$. PON1 was involved in the occurrence of kinds of inflammatory diseases including atherosclerotic, cancer and diabetes. The antioxidant function of PON1 in lung cancer tissues could affect the occurrence and progression of tumors(10). Relevant researches had proven that PON1 stimulated the growth of lung cancer cells by affecting glycolytic metabolism through antioxidant function (10). Nevertheless, anti-oxidant levels/activity would be of no benefit to KIRC. But the level of PON1 was higher in early stage than advanced stage (23). Similarly, changes in the expression and activity of PON1 caused by various factors will affect the metabolism of PON1 in tumor tissues, which will also promote the progression of the breast cancer patients(24). Besides, previous studies had proven that PON1 participated in the molecular mechanism of gastric cancer, uterine cancer and pancreatic cancer $(25,26)$.

In order to further analyze the protein expression of PON1, immunohistochemical analysis revealed that PON1 had a high expression in KIRC. GSEA is a computational method used to assess whether PON1 shows statistical differences significantly between high expression of PON1 and low expression of PON1 (27). In order to further investigate the pathophysiological mechanisms of KIRC, we carried out GSEA based on TCGA database. We employed GSEA to investigate possible signaling pathways that PON1 was involved in. Finally, we found that PON1 was involved in homologous recombination pathway. As we all know, homologous recombination is method of DNA repair. Related studies have demonstrated that the deletion of breast cancer gene $1 / 2$ can affect the occurrence and progression of breast and ovarian cancer by the way of homologous recombination (28). PON1 may influence the occurrence and development of KIRC through the mechanism of homologous recombination. Besides, we constructed PPI to explore the relationships between these proteins. As shown in results, ten genes were significantly associated with PON1, including LPA, APOC3, APOB, APOE, CLU, LCAT, APOA2, CETP and PON3. 
Relevant research had demonstrated that these genes play a significant role in tumor progression. For example, PON3 had a low expression in hepatocellular carcinoma and ovarian serous papillary carcinomas $(29,30)$. Besides, it was reported that LPA acted a pivotal part in the progression of pancreatic cancer and had relationship with certain types of cancer (31). Through the above data, interactions between PON1 and these genes revealed that PON1 might have a potential impact on the progression of KIRC. Nomogram, serving as a prognostic model, is widely used to assess the prognosis of a certain disease $(32,33)$. In this article, we conducted the nomogram based on PON1 and clinical features.

Overall, this study confirmed that PON1 could be used as an independent prognostic factor for patients with KIRC. Throughout the full article, the advantage is that it is the first time to analyze the mechanism of PON1 in KIRC. Besides, we performed validation on an independent cohort of patients by GSE14994. In addition, homologous recombination signaling pathway may be an important pathway for PON1 to regulate KIRC. There are limitations to the current study. There are few relatively studies enrichment pathways associated with KIRC in this study. Through Immunoassay results, we found that there were no correlations between PON1 and immunity. The molecular mechanism of PON1 in KIRC is not well understood. Moreover, the expression of PON1 lacked proteomic validation. Due to the lack of actual clinical data, the nomogram of this study lacked external data validation.

\section{Conclusions}

All in all, our study and the above experiments proved that PON1 could be an independent prognostic factor for patients with KIRC, and the high expression of PON1 was significantly correlated with poor prognosis. Homologous recombination was verified to be a PON1-related signaling pathway. Besides, PON1 was not associated with MSI, TMB, Neoantigen and immunity. However, the molecular mechanism of PON1 in renal clear cell carcinoma remains to be further elucidated. To further verify our findings, more data about clinical patients were required. 


\section{Ethics approval and consent to participate}

The study was approved by the Institutional Research Ethics Committees of Affiliated Hospital of Nantong University.

\section{Consent for publication}

Not applicable.

\section{Competing interests}

None declared.

\section{Funding}

None declared.

\section{Authors' Contributions}

CXJ: Protocol/project development; XYZ: Data collection or management; XYL: Data analysis; JL: Data analysis and manuscript revision; HH: Manuscript writing/editing.

\section{Acknowledgements}

We would like to thank the researchers and study participants for their contributions.

\section{Availability of data and material}

The RNA-sequencing data and corresponding clinical information were downloaded from the Cancer Genome Atlas (TCGA) database (https://portal.gdc.cancer.gov/). 


\section{References}

1. Siegel RL, Miller KD, Fuchs HE, Jemal A. Cancer Statistics, 2021. CA: a cancer journal for clinicians. 2021;71(1):7-33.

2. Li QY, Li N, Huang QB, Luo YK, Wang BJ, Guo AT, et al. Contrast-enhanced ultrasound in detecting wall invasion and differentiating bland from tumor thrombus during robot-assisted inferior vena cava thrombectomy for renal cell carcinoma. Cancer imaging : the official publication of the International Cancer Imaging Society. 2019;19(1):79.

3. Morris MR, Latif F. The epigenetic landscape of renal cancer. Nature reviews Nephrology. 2017;13(1):47-60

4. Rusek M, Pastryk JE, Bełtowski J. Modulation of paraoxonase 1 (PON1) activity and protein $\mathrm{N}$-homocysteinylation by bisphosphonates in rats. Chemico-biological interactions. 2016;259(Pt B):401-6.

5. Mackness MI, Arrol S, Durrington PN. Paraoxonase prevents accumulation of lipoperoxides in low-density lipoprotein. FEBS letters. 1991;286(1-2):152-4.

6. Tward A, Xia YR, Wang XP, Shi YS, Park C, Castellani LW, et al. Decreased atherosclerotic lesion formation in human serum paraoxonase transgenic mice. Circulation. 2002;106(4):484-90.

7. Deroo LA, Bolick SC, Xu Z, Umbach DM, Shore D, Weinberg CR, et al. Global DNA methylation and one-carbon metabolism gene polymorphisms and the risk of breast cancer in the Sister Study. Carcinogenesis. 2014;35(2):333-8.

8. Ikeda Y, Suehiro T, Arii K, Kumon Y, Hashimoto K. High glucose induces transactivation of the human paraoxonase 1 gene in hepatocytes. Metabolism: clinical and experimental. 2008;57(12):1725-32.

9. Ito T, Yasue H, Yoshimura M, Nakamura S, Nakayama M, Shimasaki Y, et al. Paraoxonase gene Gln192Arg (Q192R) polymorphism is associated with coronary artery spasm. Human genetics. 2002;110(1):89-94.

10. Aldonza MBD, Son YS, Sung HJ, Ahn JM, Choi YJ, Kim YI, et al. Paraoxonase-1 (PON1) induces metastatic potential and apoptosis escape via its antioxidative function in lung cancer cells. Oncotarget. 2017;8(26):42817-35.

11. Wang C, Liang C. MSIpred: a python package for tumor microsatellite instability classification from tumor mutation annotation data using a support vector machine. Scientific reports. 2018;8(1):17546.

12. Miao Y, Wang J, Li Q, Quan W, Wang Y, Li C, et al. Prognostic value and immunological role of PDCD1 gene in pan-cancer. International immunopharmacology. 2020;89:107080.

13. Wood M, Paralkar M, Paralkar M, Nguyen A, Struck A, Ellrott K, et al. Population-level distribution and putative immunogenicity of cancer neoepitopes. BMC cancer. 2018;18(1):414.

14. Li T, Fan J, Wang B, Traugh N, Chen Q, Liu JS, et al. TIMER: A Web Server for Comprehensive Analysis of Tumor-Infiltrating Immune Cells. Cancer research. 2017;77(21):e108-e10.

15. Wang $\mathrm{H}$, Wang MS, Wang $Y$, Huang $Y Q$, Shi JP, Ding ZL, et al. Prognostic value of immune related genes in lung adenocarcinoma. Oncology letters. 2020;20(5):259.

16. Yoshihara K, Shahmoradgoli M, Martínez E, Vegesna R, Kim H, Torres-Garcia W, et al. Inferring tumour purity and stromal and immune cell admixture from expression data. Nature communications. 2013;4:2612.

17. Liu S, Wang Y, Miao C, Xing Q, Wang Z. High expression of CDCA7 predicts poor prognosis 
for clear cell renal cell carcinoma and explores its associations with immunity. Cancer cell international. 2021;21(1):140.

18. Jonasch E, Gao J, Rathmell WK. Renal cell carcinoma. BMJ (Clinical research ed). 2014;349:g4797.

19. Schwaederle M, Zhao M, Lee JJ, Eggermont AM, Schilsky RL, Mendelsohn J, et al. Impact of Precision Medicine in Diverse Cancers: A Meta-Analysis of Phase II Clinical Trials. Journal of clinical oncology : official journal of the American Society of Clinical Oncology. 2015;33(32):3817-25.

20. Yang W, Yoshigoe K, Qin X, Liu JS, Yang JY, Niemierko A, et al. Identification of genes and pathways involved in kidney renal clear cell carcinoma. BMC bioinformatics. 2014;15 Suppl 17(Suppl 17):S2.

21. Ahmed AM. Correlation of Paraoxonase-1 with glycated hemoglobin and lipid profile among Sudanese diabetic patients. Pakistan journal of medical sciences. 2019;35(4):1050-4

22. Zintzaras E, Hadjigeorgiou GM. Association of paraoxonase 1 gene polymorphisms with risk of Parkinson's disease: a meta-analysis. Journal of human genetics. 2004;49(9):474-81.

23. Aldemir M, Karaguzel E, Okulu E, Gudeloglu A, Ener K, Ozayar A, et al. Evaluation of oxidative stress status and antioxidant capacity in patients with renal cell carcinoma. Central European journal of urology. 2015;68(4):415-20.

24. Pan X, Huang L, Li M, Mo D, Liang Y, Liu Z, et al. The Association between PON1 (Q192R and L55M) Gene Polymorphisms and Risk of Cancer: A Meta-Analysis Based on 43 Studies. BioMed research international. 2019;2019:5897505.

25. Arioz DT, Camuzcuoglu H, Toy H, Kurt S, Celik H, Erel O. Assessment of serum paraoxonase and arylesterase activity in patients with endometrial cancer. European journal of gynaecological oncology. 2009;30(6):679-82.

26. Akçay MN, Yilmaz I, Polat MF, Akçay G. Serum paraoxonase levels in gastric cancer. Hepato-gastroenterology. 2003;50 Suppl 2:cclxxiii-cclxxv.

27. Subramanian A, Kuehn H, Gould J, Tamayo P, Mesirov JP. GSEA-P: a desktop application for Gene Set Enrichment Analysis. Bioinformatics (Oxford, England). 2007;23(23):3251-3.

28. Romeo M, Pardo JC, Martínez-Cardús A, Martínez-Balibrea E, Quiroga V, Martínez-Román S, et al. Translational Research Opportunities Regarding Homologous Recombination in Ovarian Cancer. International journal of molecular sciences. 2018;19(10).

29. Choi JK, Choi JY, Kim DG, Choi DW, Kim BY, Lee KH, et al. Integrative analysis of multiple gene expression profiles applied to liver cancer study. FEBS letters. 2004;565(1-3):93-100.

30. Santin AD, Zhan F, Bellone S, Palmieri M, Cane S, Bignotti E, et al. Gene expression profiles in primary ovarian serous papillary tumors and normal ovarian epithelium: identification of candidate molecular markers for ovarian cancer diagnosis and therapy. International journal of cancer. 2004;112(1):14-25.

31. Chen J, Li H, Xu W, Guo X. Evaluation of serum ATX and LPA as potential diagnostic biomarkers in patients with pancreatic cancer. BMC gastroenterology. 2021;21(1):58.

32. Cho JK, Lee GJ, Yi KI, Cho KS, Choi N, Kim JS, et al. Development and external validation of nomograms predictive of response to radiation therapy and overall survival in nasopharyngeal cancer patients. European journal of cancer (Oxford, England : 1990). 2015;51(10):1303-11.

33. Wang X, Chen K, Wang Z, Xu Y, Dai L, Bai T, et al. Using Immune-Related Long Non-coding Ribonucleic Acids to Develop a Novel Prognosis Signature and Predict the Immune Landscape of 


\section{Figure legends}

Figure 1. The expression of PON1 in KIRC tissues; (A) PON1 is expressed differently in various cancers from TCGA database; (B)(C) PON1 expression level difference between normal tissue and the KIRC; (D) Influence of the difference of PON1 expression on the OS of patients with KIRC

Figure 2. Correlation between the expression of PON1 and clinical factors. (A) age; (B) gender; (C) grade; (D) M; (E) N; (F) race; (G) stage; (H) T

Figure 3. The expression of PON1 in normal renal tissue and kidney tissue. IHC staining exhibited the expression of PON1 is high in normal renal tissue and low, medium, high in kidney tissue.

Figure 4. PON1 could be a prognostic predictor for patients with KIRC; (A) Univariate cox regression analysis of clinicopathologic variables and PON1; (B) Multivariate cox regression analysis of clinicopathologic variables and PON1; (C) the nomogram which analyzes the overall survival of patients based on the relevant clinicopathological variables and PON1.

Figure 5. Enrichment plots from gene set enrichment analysis (GSEA). results about homologous recombination (A). Correlation between PON1 and MSI (B); PPI network (C); Correlation between PON1 and TMB (D); Correlation between PON1 and neoantigen (E).

Figure 6. The immune infiltrations and tumor microenvironment. (A) the relationship between PON1 expression and immune infiltrations which includes B cell, CD4 cell, CD8 cell, Timer Neutrophil, Timer Macrophage and Dendritic; (B) the relationship between PON1 and tumor microenvironment.

Figure 7. Expression of immune checkpoint molecules and immune cells in various tumors. (A) Expression of immune checkpoint molecules in various tumors; (B) Expression of immune cells in various tumors. 
Figures

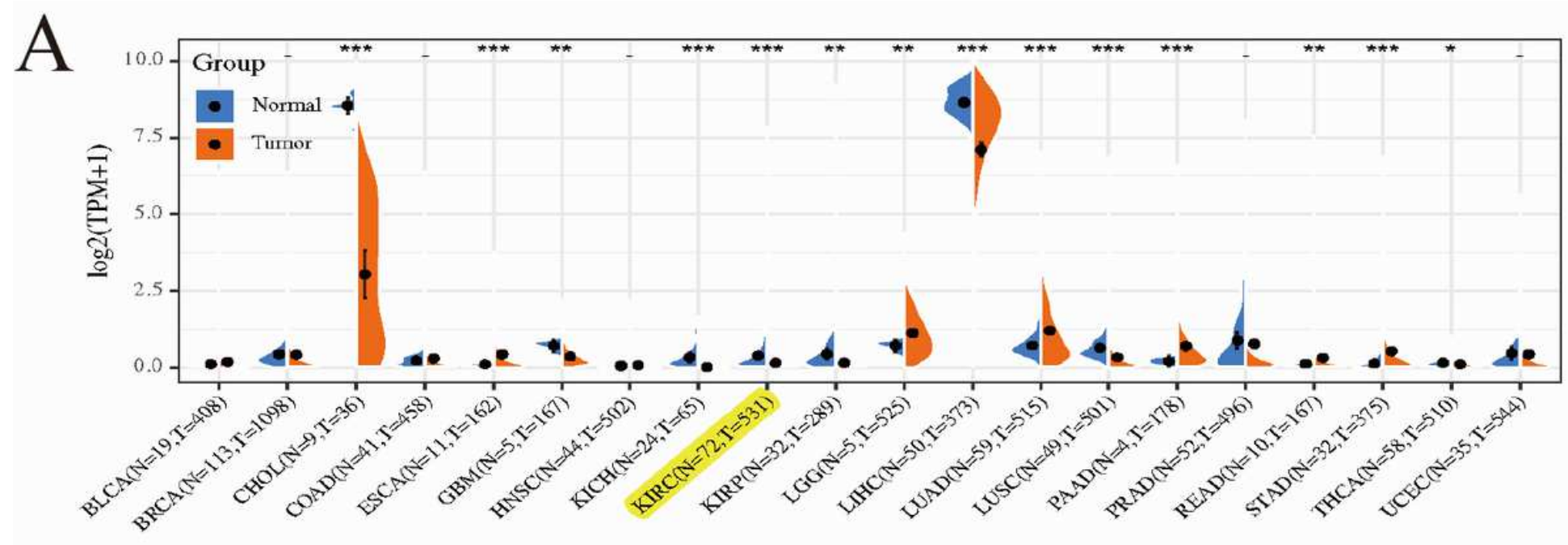

B
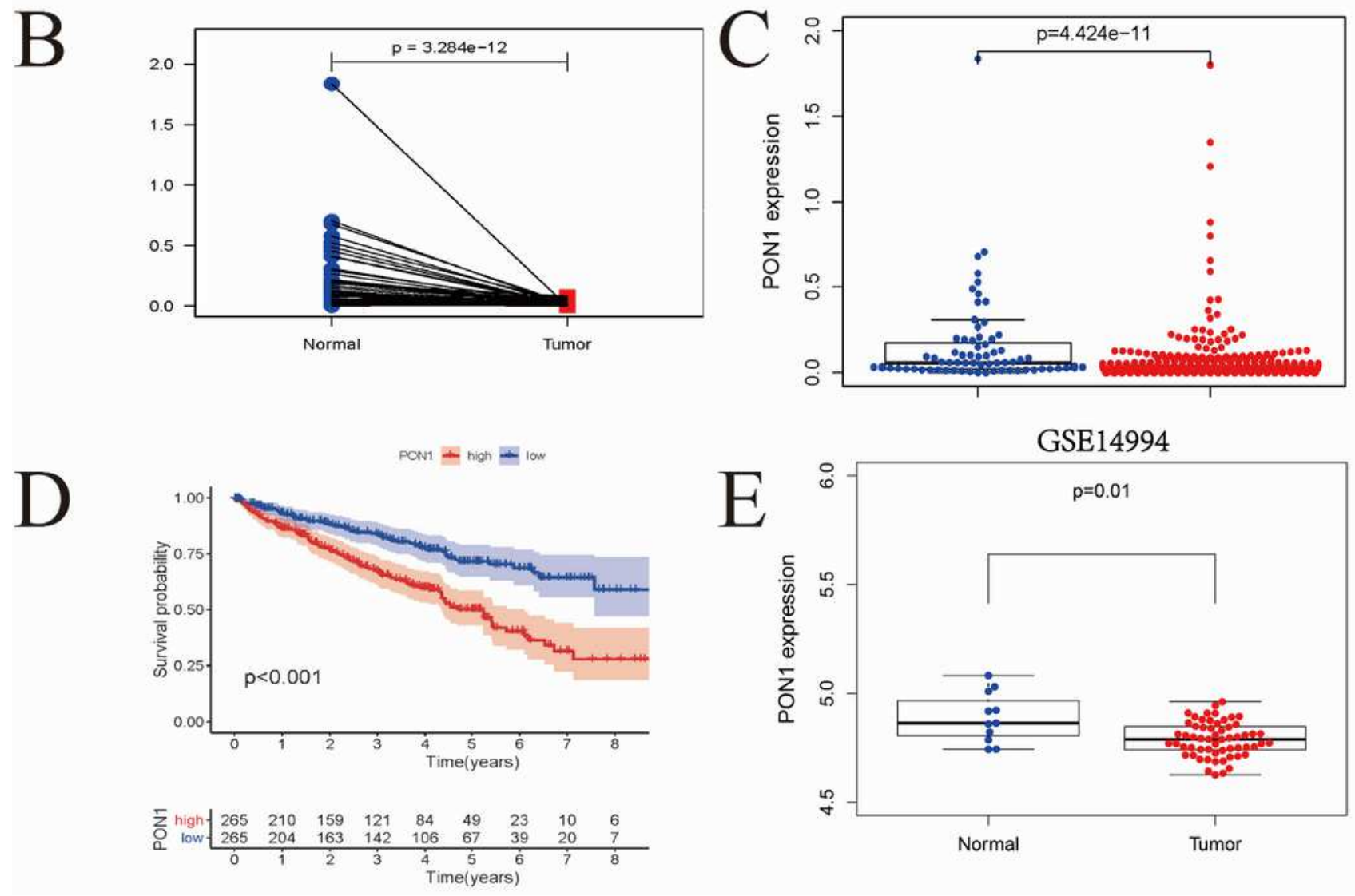

Figure 1

The expression of PON1 in KIRC tissues; (A) PON1 is expressed differently in various cancers from TCGA database; (B)(C) PON1 expression level difference between normal tissue and the KIRC; (D) Influence of the difference of PON1 expression on the OS of patients with KIRC 


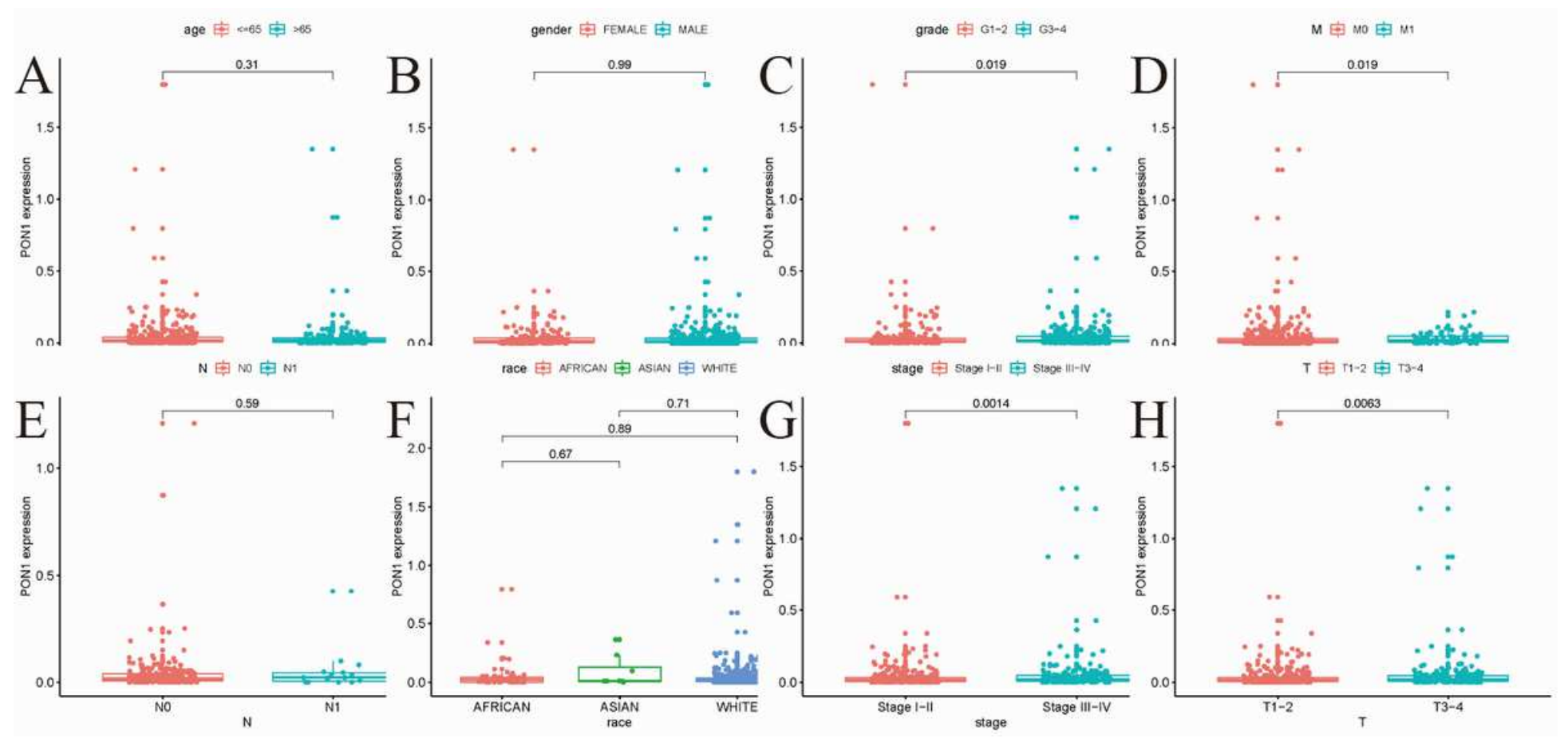

Figure 2

Correlation between the expression of PON1 and clinical factors. (A) age; (B) gender; (C) grade; (D) M; (E) $\mathrm{N} ;(\mathrm{F})$ race; $(\mathrm{G})$ stage; $(\mathrm{H}) \mathrm{T}$

Positive

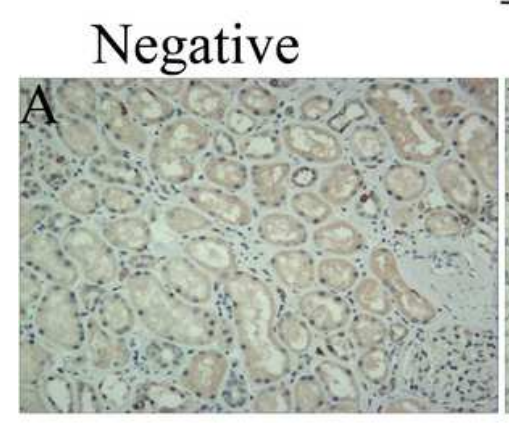

Normal

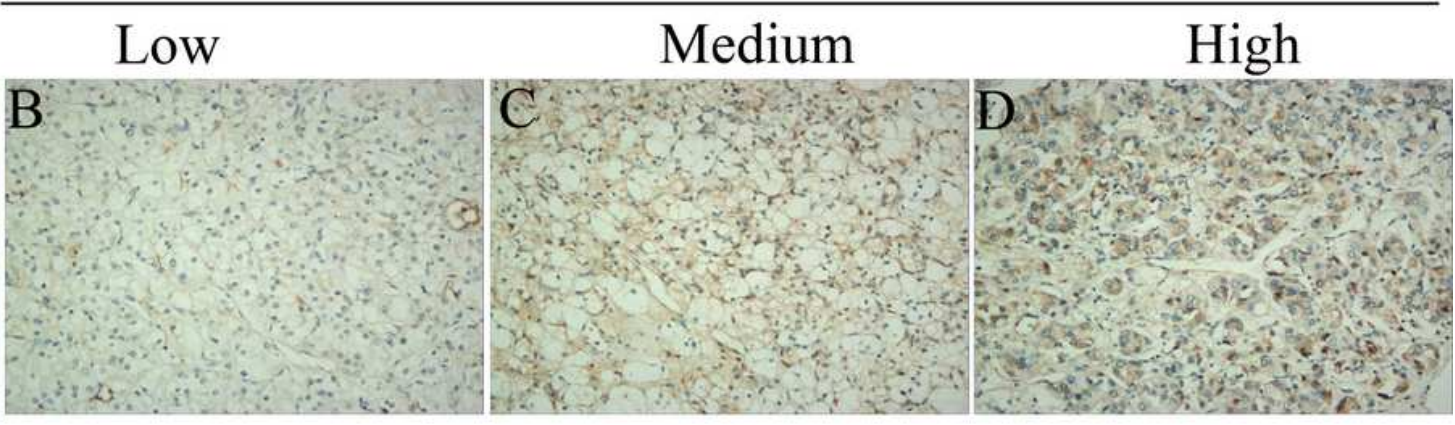

Tumor

Figure 3

The expression of PON1 in normal renal tissue and kidney tissue. IHC staining exhibited the expression of PON1 is high in normal renal tissue and low, medium, high in kidney tissue. 


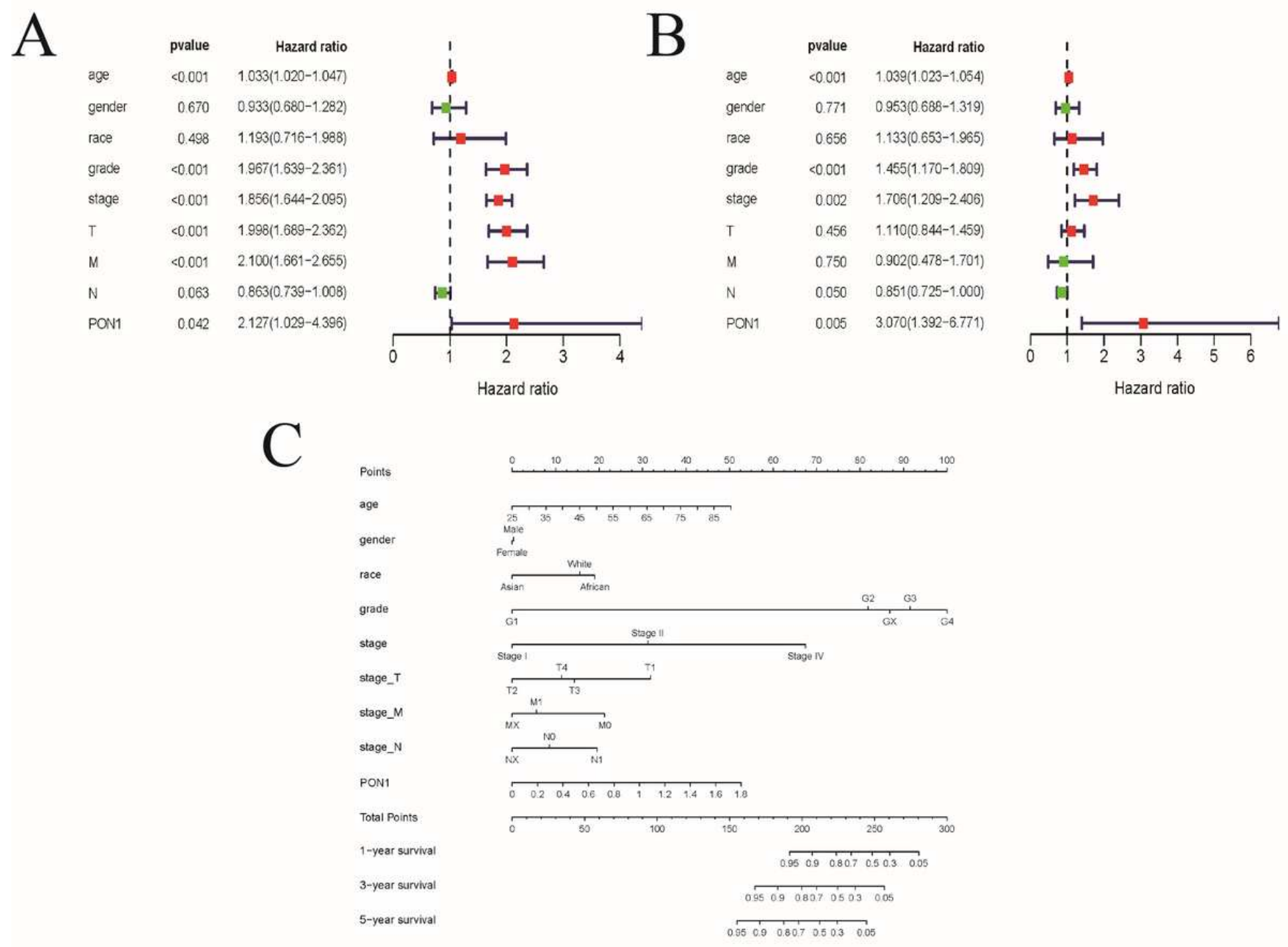

Figure 4

PON1 could be a prognostic predictor for patients with KIRC; (A) Univariate cox regression analysis of clinicopathologic variables and PON1; (B) Multivariate cox regression analysis of clinicopathologic variables and PON1; (C) the nomogram which analyzes the overall survival of patients based on the relevant clinicopathological variables and PON1. 
A

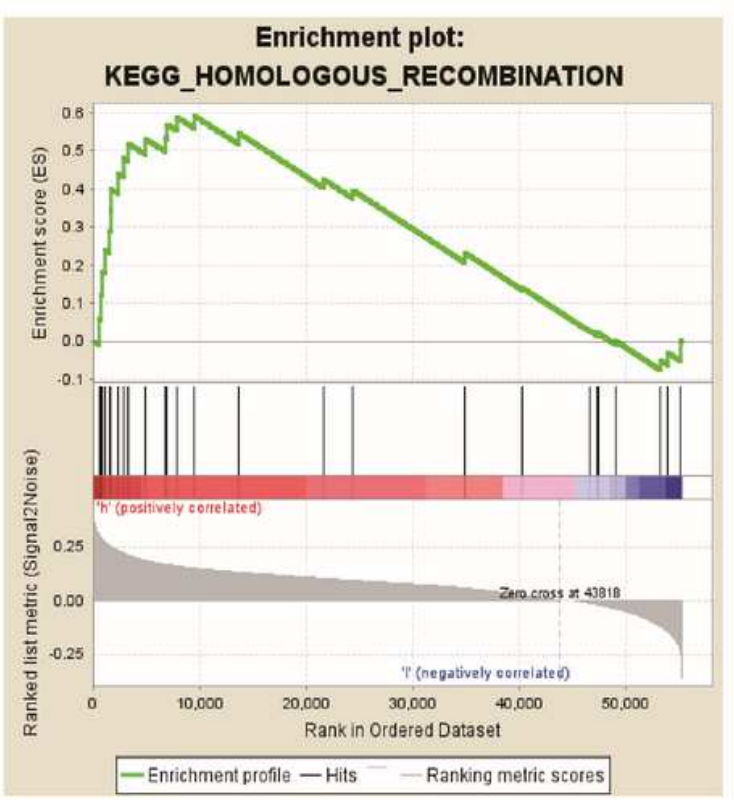

B
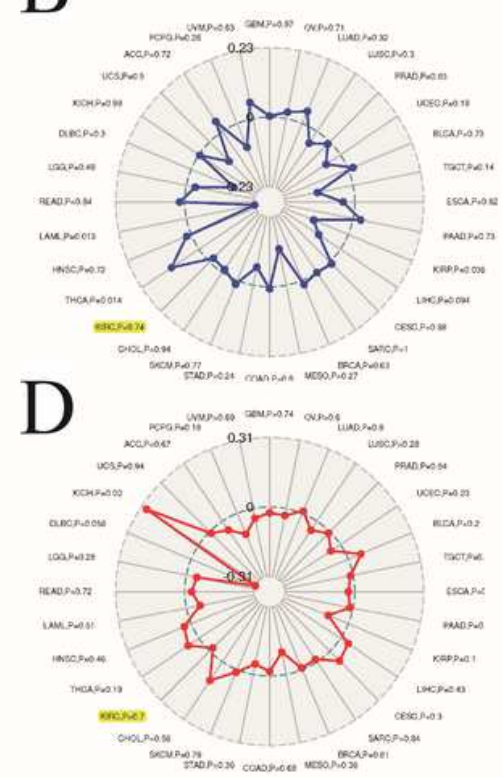

$\mathrm{C}$
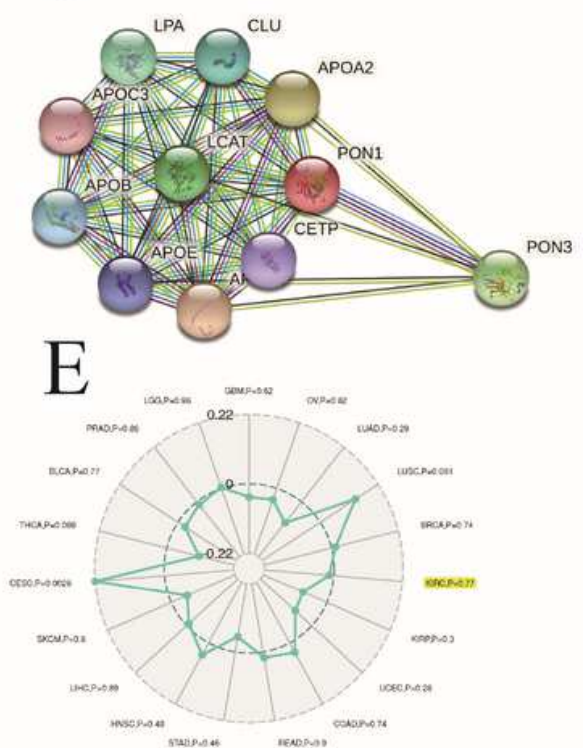

Figure 5

Enrichment plots from gene set enrichment analysis (GSEA). results about homologous recombination (A). Correlation between PON1 and MSI (B); PPI network (C); Correlation between PON1 and TMB (D); Correlation between PON1 and neoantigen (E).
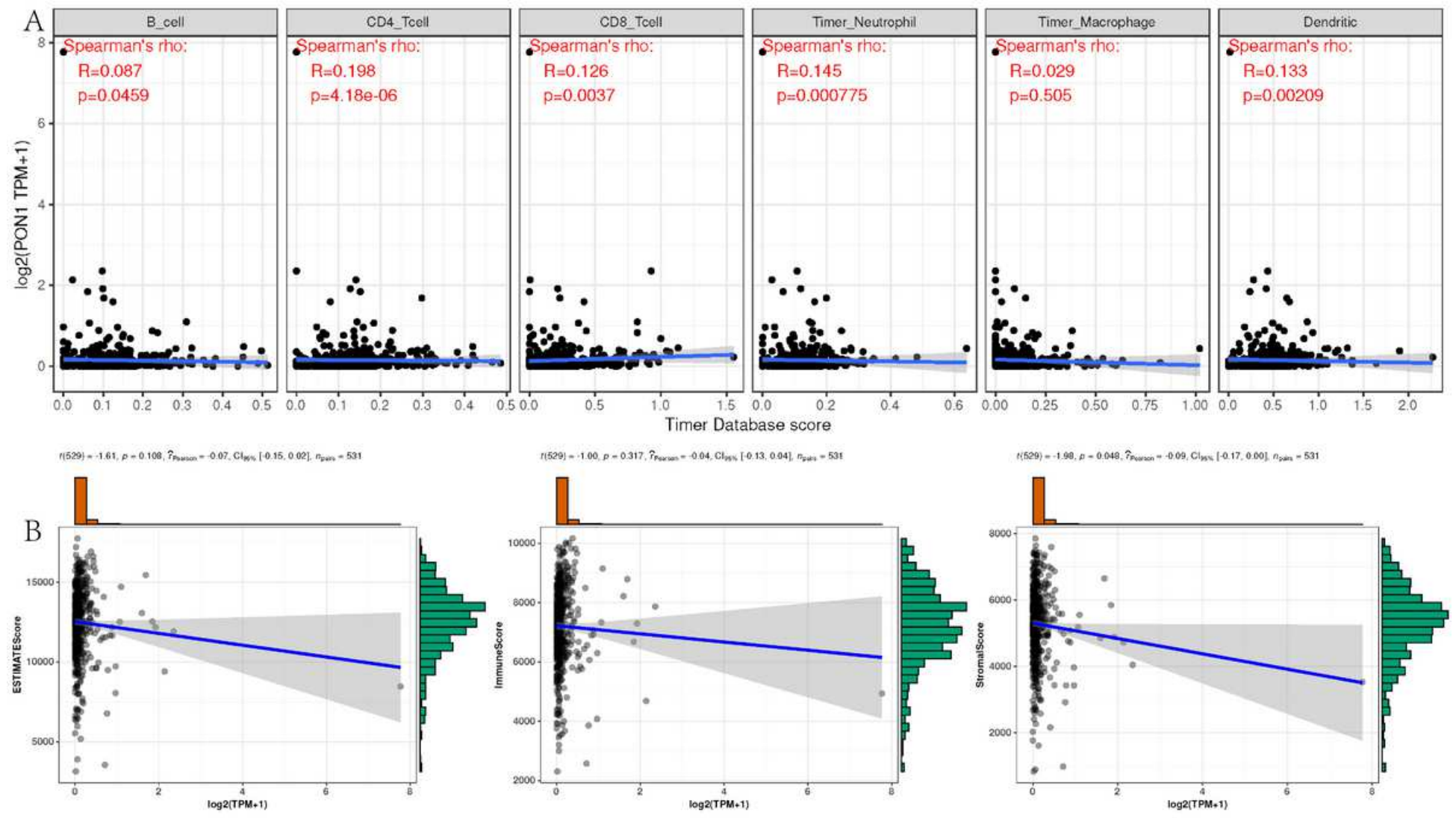

Figure 6 
The immune infiltrations and tumor microenvironment. (A) the relationship between PON1 expression and immune infiltrations which includes B cell, CD4 cell, CD8 cell, Timer Neutrophil, Timer Macrophage and Dendritic; (B) the relationship between PON1 and tumor microenvironment.
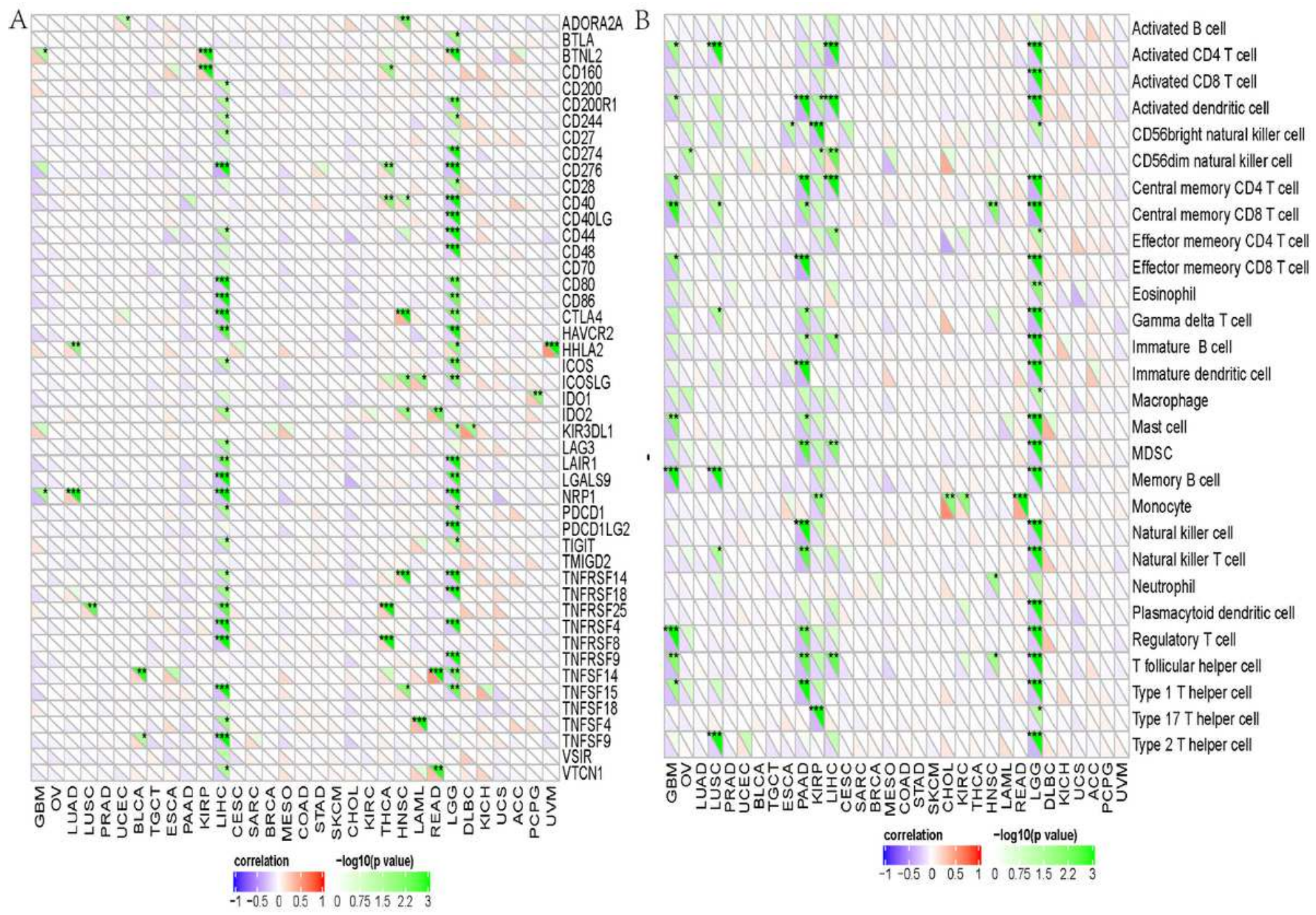

Figure 7

Expression of immune checkpoint molecules and immune cells in various tumors. (A) Expression of immune checkpoint molecules in various tumors; (B) Expression of immune cells in various tumors 